\title{
Matrix Metalloproteinase-2 Promoter Genotype as a Marker of Cutaneous T-Cell Lymphoma Early Stage
}

\author{
Anna Vasku, ${ }^{1}$ Julie Bienertova Vasku, ${ }^{1}$ Miroslav Nečas, ${ }^{2}$ and Vladimir Vasku \\ ${ }^{1}$ Department of Pathological Physiology, Faculty of Medicine, Masaryk University, University Campus Bohunice, \\ A18, Kamenice 5, 62500 Brno, Czech Republic \\ ${ }^{2}$ First Department of Dermatovenerology, St. Ann's Faculty Affiliated Hospital, Masaryk University, 65691 Brno, Czech Republic
}

Correspondence should be addressed to Anna Vasku, avasku@med.muni.cz

Received 22 January 2010; Revised 29 April 2010; Accepted 10 May 2010

Academic Editor: Peter J. Oefner

Copyright (C) 2010 Anna Vasku et al. This is an open access article distributed under the Creative Commons Attribution License, which permits unrestricted use, distribution, and reproduction in any medium, provided the original work is properly cited.

\begin{abstract}
The aim of the study was to investigate the DNA polymorphic genotype in MMP-2 promoter gene as a potential candidate region for the development of the cutaneous T-cell lymphoma (CTCL) and/or its progression. A total of 89 Czech patients with CTCL (including 23 patients with large plaque parapsoriasis) were compared to 198 controls of similar age and sex distribution, without personal or family history of chronic skin diseases and without personal history of malignancy. The three selected polymorphisms in the promoter of MMP-2 gene $(-1575 \mathrm{G} / \mathrm{A},-1306 \mathrm{C} / \mathrm{T}$, and $-790 \mathrm{~T} / \mathrm{G})$ were determined using the PCR-based methodology with RFLP. In our cohort, the associated GGCCTT MMP-2 promoter genotype was highly significantly more frequent in CTCL-Ia stage patients compared to patients with parapsoriasis, the tests having high sensitivity and specificity $(78 \%, 83 \%$, resp.). To conclude, use of associated MMP-2 promoter genotype as a DNA marker might make it possible to distinguish between the patients with parapsoriasis and those with CTCL stage Ia, which could substantially improve possibilities of clinical diagnostics, therapy design, and prognosis of this serious condition in the early stages.
\end{abstract}

\section{Introduction}

The cutaneous T-cell lymphoma is characterized by skin infiltrating and/or circulating malignant cells displaying a CD4+CD7- phenotype in the majority of cases [1]. CD4+ T lymphocytes from a nonmalignant cloned human T-cell line have been recently reported to produce a $92-\mathrm{kD}$ gelatinase (MMP 9). Apart from production of this $92-\mathrm{kD}$ gelatinase, malignant T-cell clones were reported to produce reduced amounts of a $72 \mathrm{kD}$ gelatinase (MMP 2) [1].

Generally, expression and production of matrix metalloproteinases (MMPs) is associated with advanced-stage tumour disease and MMPs are known to contribute to tumour progression, invasion, and formation of metastases. In patients with mycosis fungoides (MF), the expression of MMP-2 and MMP-9 mRNAs was observed to be significantly upregulated in advanced stages, the expression of either MMP-2 and/or MMP-9 mRNAs consistently rising with advancing disease [2]. V. Apart from their production in MF cells, the MMP-2 and/or MMP-9 mRNAs were found to be expressed by some stromal cell populations (microvascular endothelial cells, fibroblasts, macrophages), which might be suggestive of the involvement of these cells in the process of tumor invasion. Only weak positivity of the MMP-2 mRNA in microvascular endothelial cells was reported in the control skin samples [2-4], which might be of some importance for further establishment of MMP-2 as a novel clinical marker.

The large repertoire of cells inside the tumour tissue seems to be involved in the process of upregulation of MMP2 expression level during CTCL progression. Therefore, all the factors influencing MMP-2 gene expression, especially transcription factors, can participate in the process of development of CTCL. The rationale for the study is that variability in the MMP-2 gene promoter can have direct impact on binding of transcription factors, for example, by changing the affinity for given proteins.

MMP-2 gene is located at 16q13. Based on results of comparative genomic hybridization and transcriptomic data, the $16 \mathrm{p}$ region was identified as a target region for CTCLassociated gene aberrations [5]. 
There is a general consensus that MMP-mediated proteolysis is essential for cancer progression and that certain MMPs represent important targets for intervention, effective, and selective inhibition, however, pharmacological intervention in terms of these MMPs remains a major challenge in drug development $[6,7]$. Interindividual variability in the MMP genes might also play an important part in investigation of pharmacogenetic aspects of the MMP-modulated therapy.

The aim of the study was to associate DNA polymorphic genotypes in MMP-2 promoter gene with general risk for CTCL development and/or its severity (stages).

\section{Material and Methods}

2.1. Subjects. A total of 89 patients with CTCL (including 23 patients with large plaque parapsoriasis), diagnosed and treated at the 1st Department of Dermatology of St Ann's Faculty Hospital Brno (55 men and 34 women, median age $62 \mathrm{y}$, range 26-101 years) were compared to 198 controls (133 men and 65 women, median age 58 y, age range 26-80) without personal or family history of chronic skin diseases and without personal history of malignancy.

The patients with CTCL were classified according to Tumor Node Metastasis (TNM) Classification for Cutaneous T-Cell Lymphoma according to Kashani-Sabet et al. 2001 [8].

In CTCL men, $50 \%$ were treated by topical steroids ( $20 \%$ in combination with phototherapy, $2 \%$ with retinoids). The CTCL women were treated by topical steroids in 55\% ( $23 \%$ in combination with phototherapy, $5 \%$ with retinoids). Phototherapy was indicated in $51 \%$ of CTCL men and in $51 \%$ of CTCL women.

This study was approved by the Committee for Ethics of Medical Experiments on Human Subjects, Faculty of Medicine, Masaryk University, Brno (no. 64/93, 1993) and was performed in adherence to the Declaration of Helsinki Guidelines. Participants gave their written informed consent which has been archived.

2.2. Genotyping. Genomic DNA was isolated from peripheral leukocytes by a standard technique using proteinase $\mathrm{K}$.

Three MMP-2 promoter polymorphisms $(-1575 \mathrm{G} / \mathrm{A}$, $-1306 \mathrm{C} / \mathrm{T}$ and $-790 \mathrm{~T} / \mathrm{G})$ were identified by PCR method and restriction analysis according to standard protocols.

Polymorphism -1575G/A MMP-2 was detected by PCR using sense $5^{\prime}$-ACT GAC TCT GGA AAG TCA GAG CA$3^{\prime}$ and antisense $5^{\prime}$-GGC ACA GGG TGA GGG GAT GG$3^{\prime}$ primers with a PCR product of $269 \mathrm{bp}$. After restriction analysis with Tsp45I ( $\downarrow$ GT G/C AC, New England Biolabs Inc., USA) at $65^{\circ} \mathrm{C}$ for $4 \mathrm{~h}$, three genotypes could be distinguished in UV light at 2\% agarose Serva with ethidium bromide: GG (113 and 156 bp), GA (113, 156 and $269 \mathrm{bp}$ ), and AA (269 bp).

Polymorphism -1306C/T MMP-2 was detected by PCR using sense $5^{\prime}$-CTT CCT AGG CTG GTC CTT ACT GA$3^{\prime}$ and antisense $5^{\prime}$-CTG AGA CCT GAA GAG CTA AAGAGC T-3' primers with a PCR product of $193 \mathrm{bp}$. After restriction analysis with XspI (C\TAG, Takara, Japan) at $37^{\circ} \mathrm{C}$ for $4 \mathrm{~h}$, three genotypes could be distinguished: CC (188 bp), CT (188, 162 and $26 \mathrm{bp}$ ), and TT (162 and $26 \mathrm{bp}$ ).

Polymorphism -790T/G MMP-2 was detected by PCR using sense $5^{\prime}$-GGG TCT TTG TGA CCT CGA TC-3' and antisense $5^{\prime}$-GGT AAA ATG AGG CTG AGA CCT G$3^{\prime}$ primers with a $\mathrm{PCR}$ product of $118 \mathrm{bp}$. After restriction analysis with PvuI (CGAT/CG, MBI Fermentas, Lithuania) at $37^{\circ} \mathrm{C}$ for $3 \mathrm{~h}$, three genotypes could be observed in UV light at 3\% Metaphor agarose with ethidium bromide: TT (118bp), GT (118 and 19 + 99 bp), and GG (19+99 bp) [9].

2.3. Statistical Analyses. In all investigated cohorts, distributions of genotype and allelic frequencies and their differences were calculated using $\chi^{2}$ tests. Consistency of genotype frequencies with the Hardy-Weinberg equilibrium was tested using $\chi^{2}$ test on a contingency table of observed versus predicted genotype frequencies.

Odds ratio (OR) and 95\% confidence interval were calculated to estimate the risks related to detected polymorphisms. To calculate the significance of OR, Fisher's exact test was used. Sensitivity and specifity of results were calculated by Clinical Calculator 1 (http://faculty.vassar.edu/ lowry/clin1.html). The Holm's test was employed for appropriate correction to multiple comparisons. The program package Statistica v. 8.0 (Statsoft Inc., Tulsa, OK) was used.

\section{Results}

3.1. Case-Control Study. No significant differences in casecontrol analysis, neither in genotype distributions or allelic frequencies of all investigated MMP-2 polymorphisms were observed, when performing the analyses separately for the individual polymorphisms (Table 1). Similarly, no significant differences were observed between men and women, neither in the CTCL nor control cohort (data not shown).

All the three polymorphisms were found to be in linkage disequilibrium. When investigating the frequency of associated genotype (GGCCTT), that is, the most frequent in the general population, no differences between patients with CTCL and control subjects were observed (37 patients with GGCCTT/52 patients with other genotype versus 100 control subjects with GGCCTT/98 control subjects with other genotype, $P=.101)$. Similarly, no differences in the frequencies of GGCCTT genotype were observed between men and women, neither in the CTCL cohort nor the controls.

3.2. Case-Case Study. However, highly significant differences were observed when comparing the frequencies of the GGCCTT associated MMP-2 genotypes between the specific stages of CTCL. Based on our results, the GGCCTT MMP2 promoter genotype is significantly more frequent in CTCL-Ia stage patients compared to all other CTCL groups including parapsoriasis (Table 2). All comparisons retained their significance after appropriate correction for multiple comparisons $\left(P_{\text {corr }}\right)$. The GGCCTT genotype was observed to be the most sensitive and specific marker to distinguish between CTCL patients with stage IA and the patients 
TABLE 1: Three promoter MMP-2 gene polymorphisms: patients with CTCL versus control subjects.

\begin{tabular}{|c|c|c|c|c|c|c|c|}
\hline & \multicolumn{7}{|c|}{$-1575 / G$} \\
\hline Genotypes and alleles & GG (\%) & GA (\%) & AA (\%) & $P_{g}$ & G & A & $P_{a}$ \\
\hline CTCL $(N=89)$ & $48(54)$ & $37(42)$ & $4(4)$ & \multirow{2}{*}{0.931} & 0.747 & 0.253 & \multirow{2}{*}{0.804} \\
\hline \multirow[t]{2}{*}{ Controls $(N=198)$} & $105(53)$ & $82(41)$ & $11(6)$ & & 0.737 & 0.263 & \\
\hline & \multicolumn{7}{|c|}{$-1306 \mathrm{C} / \mathrm{T}$} \\
\hline Genotypes and alleles & CC (\%) & CT (\%) & TT (\%) & \multirow{3}{*}{0.492} & $\mathrm{C}$ & $\mathrm{T}$ & \multirow{3}{*}{0.853} \\
\hline CTCL $(N=89)$ & $46(52)$ & $41(46)$ & $2(2)$ & & 0.747 & 0.253 & \\
\hline \multirow[t]{2}{*}{ Controls $(N=198)$} & $105(53)$ & $83(42)$ & $10(5)$ & & 0.740 & 0.260 & \\
\hline & \multicolumn{7}{|c|}{$-790 \mathrm{~T} / \mathrm{G}$} \\
\hline Genotypes and alleles & TT (\%) & TG (\%) & GG (\%) & \multirow{3}{*}{0.091} & $\mathrm{~T}$ & G & \multirow{3}{*}{0.258} \\
\hline $\operatorname{CTCL}(N=89)$ & $46(52)$ & $39(44)$ & $4(4)$ & & 0.736 & 0.264 & \\
\hline Controls $(N=198)$ & $100(51)$ & $73(37)$ & $25(12)$ & & 0.689 & 0.311 & \\
\hline
\end{tabular}

$P_{\mathrm{g}}=P$-value of a difference in genotype distribution

$P_{\mathrm{a}}=P$-value of a difference in allelic frequencies.

TABLE 2: Risk for GGCCTT associated genotype in MMP-2 gene promoter in different CTCL stages compared to CTCL-Ia.

\begin{tabular}{lcccr}
\hline & MMP-2-GGCCTT $(\%)$ & MMP-2-the others $(\%)$ & Odds ratio & $P$-value $\left(P_{\text {corr }}{ }^{* * *}\right)$ \\
\hline LPP $^{*}(N=23)$ & $4(17)$ & $19(83)$ & $0.07(0.01-0.28)$ & $.00004(0.0002)$ \\
CTCL-Ia $(N=23)$ & $18(77)$ & $5(23)$ & & \\
CTCL-Ib $(N=24)$ & $10(42)$ & $14(58)$ & $0.10(0.06-0.71)$ & $.01(0.02)$ \\
CTCL II $(N=11)$ & $3(25)$ & $8(75)$ & $0.10(0.02-0.55)$ & $.007(0.03)$ \\
CTCL III+IV $(N=8)$ & $2(30)$ & $6(70)$ & $0.09(0.01-0.61)$ & $.01(0.02)$ \\
Controls $(N=198)$ & $100(51)$ & $98(49)$ & $0.28(0.10-0.79)$ & $.009(0.03)$ \\
\hline
\end{tabular}

* LPP- large plaque parapsoriasis

** Odds ratio for GGCCTT in any group compared to CTCL-Ia (95\% confidential interval)

*** $P_{\text {corr }}=P$-value corrected for multiple comparison.

with parapsoriasis (sensitivity is $78 \%, 95 \% \mathrm{CI}=56-92 \%$; specifity is $83 \%, 95 \% \mathrm{CI}=60-94 \%$ ).

When evaluating the risk of GGCCTT MMP-2 genotype for development of parapsoriasis, we observed higher frequencies of the GGCCTT genotype in all other CTCL groups as well as in control subjects (Table 3 ). In this case, the differences were significant when comparing the CTCLIa patients and the control subjects.

\section{Discussion}

Early diagnosis of CTCL is an extremely important point for efficient decision-making in the treatment of CTCL. However, there is no generally accepted consensus on the range of criteria that should be employed to achieve as early diagnosis of the disease as possible [10]. The differential diagnosis between early stages of mycosis fungoides and large plaque parapsoriasis (both considered a form of CTCL) can be often difficult, especially when being based merely on evaluation of clinical and histological parameters [11]. Therefore, there is a strong need for novel markers that would make the earlier diagnosis of the disease possible; for example, CD13 (aminopeptidase N) was recently identified as an early prognostic factor in CTCL [12]. Based on our results, the variability of the MMP-2 promoter region might act as potential biomarker of different CTCL stages, especially as for the CTCL stage IA and large plaque parapsoriasis. This is to the best of our knowledge the first study of given SNPs in MMP-2 gene on CTCL cases, which was carried out on a highly static population of the South Moravia, of a Central-European Caucasian origin. Not surprisingly, it will be necessary to replicate the results of the study on larger population samples of different ethnicity to achieve a final conclusion of the importance and significance of the MMP-2 gene variability in CTCL.

Taking into account the structure of DNA in the given locus, the GGCCTT genotype in the promoter of MMP-2 gene should have a high transcription rate. Two functional SNPs in vitro in the MMP-2 promoter were recently investigated: $-1575 \mathrm{G}$ to $\mathrm{A}$, which alters binding of oestrogen receptor [13], and $-1306 \mathrm{C}$ to $\mathrm{T}$, which alters binding of Sp-1. The $\mathrm{C}$, but not $\mathrm{T}$ allele of $-1306 \mathrm{C} / \mathrm{T}$ MMP-2 polymorphism contains an Sp1 motif (CCAACC box), therefore displaying strikingly higher promoter activity in vitro [14]. The transcription factor $\mathrm{Sp} 1$ is a DNAbinding protein which interacts with a variety of gene promoters containing GC-box elements. The factor regulates the expression of a number of genes participating in multiple aspects of tumorigenesis, such as angiogenesis, cell growth, and apoptosis resistance. The Sp1 levels in untransformed 
TABLE 3: Risk for GGCCTT associated genotype in MMP-2 gene promoter in different CTCL stages compared to parapsoriasis.

\begin{tabular}{|c|c|c|c|c|}
\hline & MMP-2-GGCCTT (\%) & MMP-2-the others (\%) & Odds ratio ${ }^{*}$ & $P$-value $\left(P_{\text {corr }}{ }^{* *}\right)$ \\
\hline $\operatorname{LPP}^{*}(N=23)$ & $4(17)$ & $19(83)$ & & \\
\hline CTCL-Ia $(N=23)$ & $18(77)$ & $5(23)$ & $17.10(3.95-73.95)$ & $.00004(0.0002)$ \\
\hline CTCL-Ib $(N=24)$ & $10(42)$ & $14(58)$ & $3.39(0.88-13.08)$ & .07 \\
\hline CTCL II $(N=11)$ & $3(25)$ & $8(75)$ & $1.78(0.32-9.85)$ & .400 \\
\hline CTCL III+IV $(N=8)$ & $2(30)$ & $6(70)$ & $1.58(0.23-10.90)$ & .497 \\
\hline Controls $(N=198)$ & $100(51)$ & $98(49)$ & $4.85(1.59-14.76)$ & $.002(0.008)$ \\
\hline
\end{tabular}

* LPP - large plaque parapsoriasis

** Odds ratio for GGCCTT in any group compared to LPP (95\% confidential interval)

${ }^{* * *} P_{\text {corr }}=P$-value corrected for multiple comparison.

cells must be tightly regulated as Sp1 overexpression leads to the induction of apoptosis. Recently, Sp1 has been shown to be overexpressed in a number of human cancers, whereas its overexpression is supposed to contribute to malignant transformation [15].

Furthermore, in silico, three transcription factors could be found in the DNA sequence including the $\mathrm{T}$ allele of $-790 \mathrm{G} / \mathrm{T}$ MMP-2 polymorphism: KLF4 (Kruppel-like factor 4, S8 and Evil-ectopic viral integration site 1 encoded factor (according to the Transfac Matrix Table (MatInspector V2.2). KLF4 represents the essential transcription factor which is critical and selective for establishment of the proper barrier function of the skin [16]. KLF4 was proved to interact with beta-catenin and to inhibit Wnt signalling [17]. MMP-2 seems to be a Wnt target gene because two TCF sites typical for this gene were identified in MMP-2 gene promoter, interestingly in a close vicinity of the $-790 \mathrm{G} / \mathrm{T}$ polymorphism (at -861 and -804 positions). Wnt signalling was observed not only to induce expression of matrix metalloproteinase 2 , but also to regulate transmigration of $\mathrm{T}$ cells [18]. Wnts probably induces a T-cell phenotype capable of migration through collagen [18]. This ability is crucial for extravasation of $\mathrm{T}$ cells, which is not observed in parapsoriasis as a rule. Finally, this could explain the observed difference in variability of promoter of MMP-2 gene between the patients with parapsoriasis and CTCL-Ia stage. Last but not least, Evi1 is a highly conserved transcription factor that was shown to upregulate cell proliferation, to impair cell differentiation and also to induce malignant transformation [19].

To conclude, the frequency of the GGCCTT MMP2 genotype was the lowest in the group of patients with parapsoriasis, when compared to the control subjects. On the other hand, the efficient form of MMP-2 promoter seems to be associated with CTCl Ia stage. We propose an idea that the detection of MMP-2 promoter polymorphism might contribute to resolution between specific stages in the framework of CTCL cohort, that is, between the patients with parapsoriasis and CTCL Ia stage, whereas the test has satisfactory sensitivity and specifity. From the clinical point of view, the GGCCTT MMP-2 genotype can be considered a potential DNA marker that might be employed to assist in clinical resolution between CTCL Ia and parapsoriasis. Our study continues in newly diagnosed patients with large plaque parapsoriasis and with all stages of CTCL, examination of MMP-2 gene expression will be performed from bioptic skin lesion samples by RT-PCR method. This enables to evaluate the actual functional association between the promoter MMP-2 genotype and MMP-2 expression in the skin of patients with a different stage of CTCL. Definitely, further studies on larger population sample are necessary that could substantially complete possibilities of clinical diagnostics, individualize therapy design, and improve prognosis of the serious disease with respect to variability of MMP-2 gene.

\section{References}

[1] D. Hauzenberger, S. E. Bergström, J. Klominek, and K. G. Sundqvist, "Spectrum of extracellular matrix degrading enzymes in normal and malignant T lymphocytes," Anticancer Research, vol. 19, no. 3, pp. 1945-1952, 1999.

[2] K. Nabeshima, J. Suzumiya, M. Nagano et al., "Emmprin, a cell surface inducer of matrix metalloproteinases (MMPs), is expressed in T-cell lymphomas," Journal of Pathology, vol. 202, no. 3, pp. 341-351, 2004.

[3] A. Vacca, S. Moretti, D. Ribatti et al., "Progression of mycosis fungoides is associated with changes in angiogenesis and expression of the matrix metalloproteinases 2 and 9," European Journal of Cancer, vol. 33, no. 10, pp. 1685-1692, 1997.

[4] A. Vacca, D. Ribatti, R. Ria et al., "Proteolytic activity of human lymphoid tumor cells. Correlation with tumor progression," Developmental Immunology, vol. 7, no. 2-4, pp. 77-88, 2000.

[5] S. Hahtola, S. Tuomela, L. Elo et al., "Th1 response and cytotoxicity genes are down-regulated in cutaneous T-cell lymphoma," Clinical Cancer Research, vol. 12, no. 16, pp. 4812-4821, 2006.

[6] M. Arlt, C. Kopitz, C. Pennington et al., "Increase in gelatinase-specificity of matrix metalloproteinase inhibitors correlates with antimetastatic efficacy in a T-cell lymphoma model," Cancer Research, vol. 62, no. 19, pp. 5543-5550, 2002.

[7] A. Krüger, M. J. E. Arlt, M. Gerg et al., "Antimetastatic activity of a novel mechanism-based gelatinase inhibitor," Cancer Research, vol. 65, no. 9, pp. 3523-3526, 2005.

[8] M. Kashani-Sabet, A. McMillan, and H. S. Zackheim, "A modified staging classification for cutaneous T-cell lymphoma," Journal of the American Academy of Dermatology, vol. 45, no. 5, pp. 700-706, 2001.

[9] A. Vašků, M. Goldbergová, L. I. Hollá et al., "A haplotype constituted of four MMP-2 promoter polymorphisms ($1575 \mathrm{G} / \mathrm{A},-1306 \mathrm{C} / \mathrm{T},-790 \mathrm{~T} / \mathrm{G}$ and $-735 \mathrm{C} / \mathrm{T})$ is associated with coronary triple-vessel disease," Matrix Biology, vol. 22, no. 7, pp. 585-591, 2004. 
[10] K. N. Sarveswari and P. Yesudian, "The conundrum of parapsoriasis versus patch stage of mycosis fungoides," Indian Journal of Dermatology, Venereology and Leprology, vol. 75, no. 3, pp. 229-235, 2009.

[11] M. Santucci, A. Biggeri, A. C. Feller, D. Massi, and G. Burg, "Efficacy of histologic criteria for diagnosing early mycosis fungoides: an EORTC cutaneous lymphoma study group investigation," American Journal of Surgical Pathology, vol. 24, no. 1, pp. 40-50, 2000.

[12] C. Bernier, J.-M. Nguyen, G. Quéreux, J.-J. Renault, B. Bureau, and B. Dreno, "CD13 and TCR clone: markers of early mycosis fungoides," Acta Dermato-Venereologica, vol. 87, no. 2, pp. 155-159, 2007.

[13] S. Harendza, D. H. Lovett, U. Panzer, Z. Lukacs, P. Kühnl, and R. A. K. Stahl, "Linked common polymorphisms in the gelatinase a promoter are associated with diminished transcriptional response to estrogen and genetic fitness," The Journal of Biological Chemistry, vol. 278, no. 23, pp. 2049020499, 2003.

[14] S. J. Price, D. R. Greaves, and H. Watkins, "Identification of novel, functional genetic variants in the human matrix metalloproteinase-2 gene. Role of Sp1 in allele-specific transcriptional regulation," The Journal of Biological Chemistry, vol. 276, no. 10, pp. 7549-7558, 2001.

[15] E. Deniaud, J. Baguet, A.-L. Mathieu, G. Pagès, J. Marvel, and Y. Leverrier, "Overexpression of Sp1 transcription factor induces apoptosis," Oncogene, vol. 25, no. 53, pp. 7096-7105, 2006.

[16] J. A. Segre, C. Bauer, and E. Fuchs, "Klf4 is a transcription factor required for establishing the barrier function of the skin," Nature Genetics, vol. 22, no. 4, pp. 356-360, 1999.

[17] P. M. Evans, X. Chen, W. Zhang, and C. Liu, "KLF4 interacts with $\beta$-catenin/TCF4 and blocks $\mathrm{p} 300 / \mathrm{CBP}$ recruitment by catenin," Molecular and Cellular Biology, vol. 30, no. 2, pp. 372-381, 2010.

[18] B. Wu, S. P. Crampton, and C. C. W. Hughes, "Wnt signaling induces matrix metalloproteinase expression and regulates $\mathrm{T}$ cell transmigration," Immunity, vol. 26, no. 2, pp. 227-239, 2007.

[19] O. Fuchs, "EVI1 and its role in myelodysplastic syndrome, myeloid leukemia and other malignant diseases," Casopis Lekaru Ceskych, vol. 145, no. 8, pp. 619-624, 2006. 


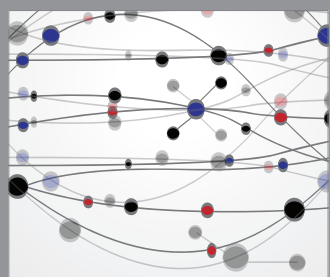

The Scientific World Journal
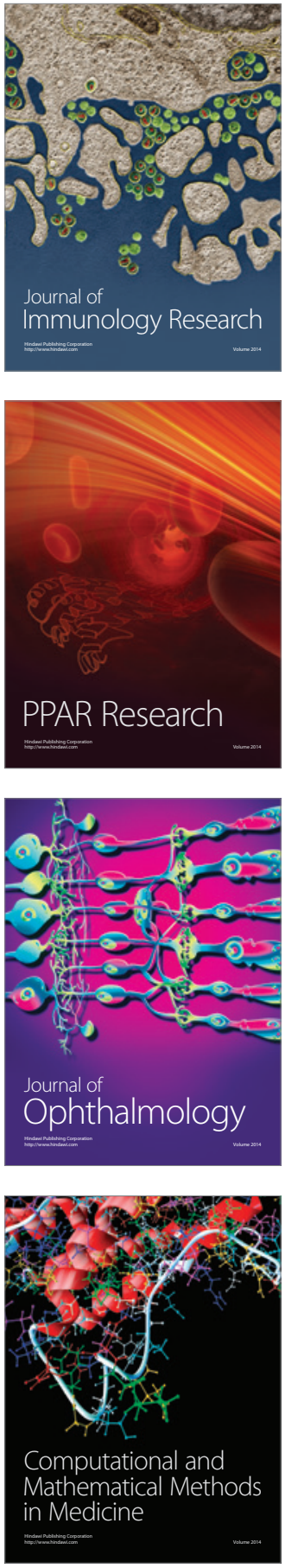

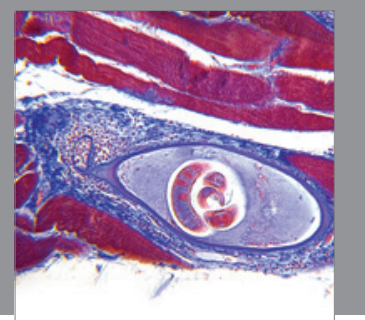

Gastroenterology

Research and Practice
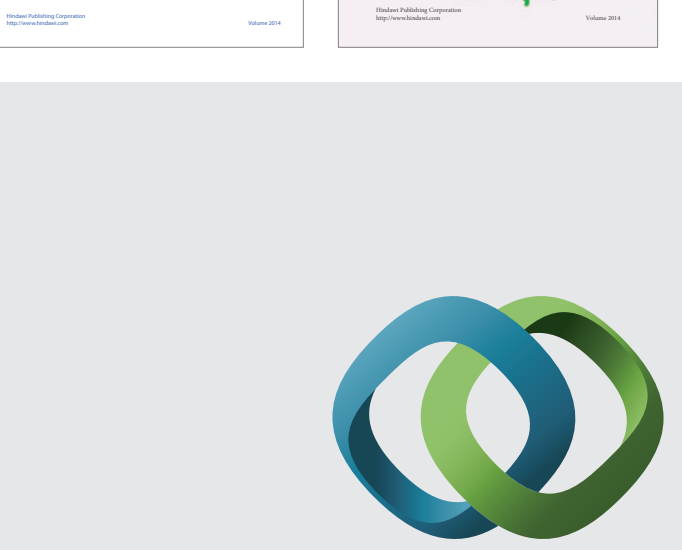

\section{Hindawi}

Submit your manuscripts at

http://www.hindawi.com
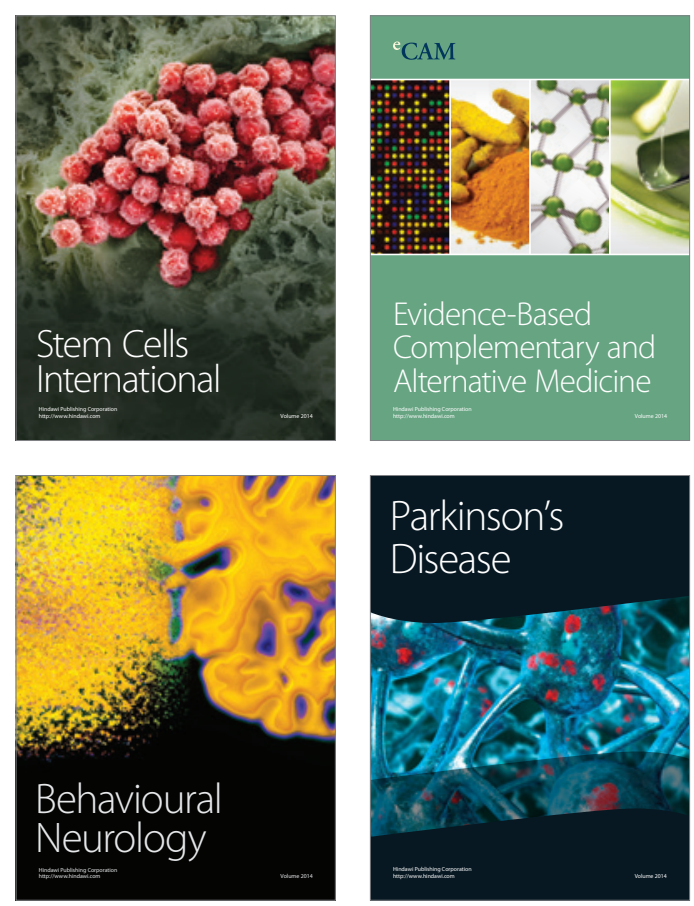

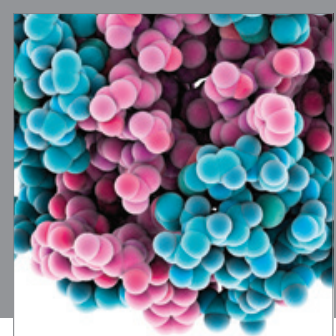

Journal of
Diabetes Research

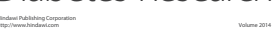

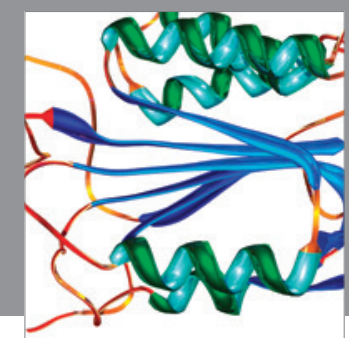

Disease Markers
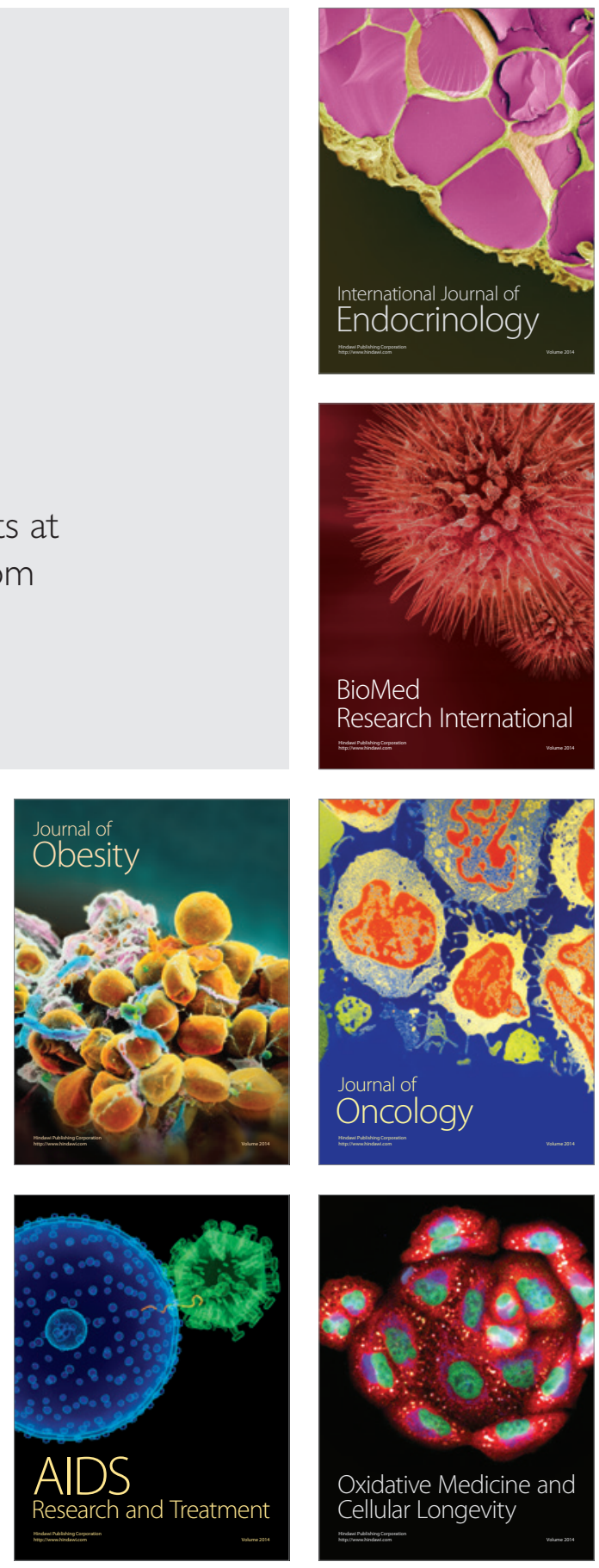